\title{
Estimación de la producción láctea del ganado Jersey usando variables temporales, intrínsecas y ambientales
}

\author{
Rodolfo WingChing-Jones ${ }^{1}$ (D) \& Juan Federico Conejo-Morales ${ }^{2}$ \\ 1. Universidad de Costa Rica, Escuela de Zootecnia, Centro de Investigación en Nutrición Animal (CINA), San José, \\ Costa Rica; rodolfo.wingching@ucr.ac.cr \\ 2. Ciudad de los Niños, Cartago, Costa Rica; federicoconejo@gmail.com
}

\author{
Recibido 20-VI-2019 • Corregido 2-X-2019 • Aceptado 21-XI-2019 \\ DOI: https://doi.org/10.22458/urj.v12i1.2792
}

\begin{abstract}
Dairy production of Jersey cattle estimation using temporary, intrinsic and environmental variables". Introduction: Production systems looking for efficient use of resources are continually predicting the dairy production behavior of production animals. Objective: To generate a mathematical prediction model of dairy production that associates climate conditions at the moment the information was collected with the intrinsic characteristics of the livestock in two production systems located at 600 and 1800 m.a.s.I. in Cartago, Costa Rica. Methods: We used a database created from 2003 to 2014 for the production system located at 600 m.a.s.l. with a total of 61 animals, while for the system established at 1 800 m.a.s.l. the information was recorded between the 2011 to 2014, with 387 animals. Results: We generated three equations under the hypothesis that the producers do not gather and record the complete information. The first one considers the interaction of environmental, temporal, and intrinsic variables of the animal, while the other two equations separate the environmental effect and the characteristics of the animal. We determined that at 600 m.a.s.l. the temperature $(-4,52)$, lactation number $(-0,99)$, the relative humidity $(-0,16)$, the moth $(-0,14)$ and lactation days $(-0,02)$ have an adverse effect on milk production, while precipitation $(0,0004)$, radiation $(0,044)$, the age of the animal $(0,084)$ and humidity and temperature index $(2,68)$ favor productivity. At the same time, at 1800 m.a.s.I. radiation $(-0,06)$, relative humidity ($0,04)$ and lactating days $(-0,03)$ decreased animal productivity, on the other hand, the assessment precipitation $(0,02)$, month $(0,07)$ and lactation number $(0,57)$ increased. Conclusion: These equations are input so that those responsible for the production systems have a way to select animals or take actions to maintain animal homeostasis.
\end{abstract}

Keywords: Relative humidity, temperature, dairycattle, Bos taurus, heat stress.
RESUMEN. Introducción: Predecir el comportamiento de la producción láctea es una actividad constante en los sistemas de producción en busca del uso eficiente de los recursos. Objetivo: Generar un modelo matemático de predicción de la producción láctea que relacione las condiciones climáticas presentes en el momento que se recolectó la información y las características intrínsecas que presentaban los semovientes en dos sistemas de producción ubicados a 600 y 1800 m.s.n.m. en la provincia de Cartago, Costa Rica. Métodos: Utilizamos bases de datos generadas desde el 2003 al 2014, en el sistema ubicado a 660 m.s.n.m. para un total de 61 animales, mientras que a 1800 m.s.n.m., la información la registramos entre 2011 al 2014, con el aporte de 387 animales. Resultados: Generamos tres ecuaciones bajo la hipótesis de que los productores no registran la información completa, la primera considera la interacción de las variables ambientales, temporales e intrínsecas del animal, mientras que las siguientes dos ecuaciones, separan el efecto ambiental y las características del animal. A 600 m.s.n.m, la temperatura $(-4,52)$, el número de lactancia $(-0,99)$, la humedad relativa $(-0,16)$, el mes $(-$ $0,14)$ y los días de lactancia $(-0,02)$ presentaron un efecto negativo hacia la producción, mientras que la precipitación $(0,0004)$, radiación $(0,044)$, la edad del semoviente $(0,084)$, y el índice de humedad y temperatura $(2,68)$, favorecieron la productividad. En cambio a 1800 m.s.n.m., la radiación $(-0,06)$, la humedad relativa $(-0,04)$ y los días de lactancia $(-0,03)$ disminuyeron la productividad del animal, por el contrario, la precipitación $(0,02)$, el mes de evaluación $(0,07)$ y el número de lactancia $(0,57)$ la aumentaron. Conclusión: Estas ecuaciones son un insumo para que los responsables de los sistemas productivos, tengan una forma de seleccionar o tomar acciones para mantener la homeostasis de los animales.

Palabras clave: Humedad relativa, temperatura ambiental, ganado lechero, Bos taurus, estrés calórico. 
Explicar el comportamiento de la producción de leche por medio de herramientas matemáticas, es una función que se repite en cualquier sistema donde se quiere predecir la productividad o explicar las fluctuaciones en la producción en miras de la optimización de los recursos. En el caso del semoviente productor de leche, se notan diferencias en su producción entre ordeños (Klopcic, Koops, \& Kuipers, 2013), días o lactancias. Fluctuaciones que se pueden relacionar, al año de parto (Quintero, Serna, Hurtado, Rosero, \& Cerón-Muñoz, 2007), mes del parto, número de partos (Castello-Umaña, Alpizar-Naranjo, Padilla-Fallas, \& Keim-San Martin, 2017), edad del animal (WingChing-Jones, Pérez, \& Salazar, 2008), a la edad que inicio la primera lactancia (CastilloBadilla, Salazar-Carranza, Murillo-Herrera, \& Romero-Zúñiga, 2013), el número de lactancia en donde se encuentran, capacidad de adaptación del semoviente (Berman, 2011), la raza (Xue, Yan, Ferris, \& Mayne, 2011), el estado de preñez (Rodríguez, Olivares, Sánchez, Alemán, \& Arece, 2013), condición corporal del animal (Roche et al., 2009), variaciones nutricionales (consumo de materia, cumplimiento de los requerimientos) (Krizsan, Sairanen, Hojer, \& Huhtanen, 2014), el estado sanitario, conteo de células somáticas (Hagnestam-Nielsen, Emanuelson, Berglund, \& Strandberg, 2009), la disponibilidad de forraje y distribución de los apartos; y las condiciones climáticas donde se encuentran los semovientes (precipitación, temperatura, humedad relativa, radiación y velocidad del viento), como las interacciones entre dos o más variables.

Dentro de los modelos o funciones matemáticas desarrolladas para explicar el comportamiento de la producción láctea, se citan los trabajos de Quintero et al. (2007), Cole, Ehrlich y Null (2012), Murphy, O’Mahony, Shalloo, French y Upton (2014) y Cuevas et al. (2018), donde tratan de predecir la producción de leche por medio de la recolección de información parcial o completa relacionada con el sistema de producción. En el caso específico para Costa Rica, se resumen los trabajos de Molina y Boschini (1979) en animales Holstein y Vargas-Leitón y SolanoPatiño (1995), Valerín (1997), Cascante (2008) y Castello-Umaña et al. (2017), en animales Jersey.

En el marco de las condiciones climáticas, se resumen experiencias del efecto del clima sobre la productividad de los animales, pero escasos modelos permiten estimar el impacto del clima sobre la producción de los animales, relación que toma relevancia con las variaciones de clima producto del cambio climático. Estas condiciones afectan de dos formas a los semovientes. De manera indirecta propicia la presencia de parásitos externos e internos (Rodríguez et al., 2013), afecta el crecimiento, desarrollo y calidad de las pasturas (Sánchez \& Soto, 1999), genera condiciones en el suelo que afectan la locomoción de los animales (WingChing-Jones, CabalcetaAguilar, \& Alvarado-Hernández, 2009) y la salud de las pezuñas (Solano et al., 2016). Por otro lado, la exposición directa a la radiación, la velocidad del viento, variaciones en el índice de temperatura y humedad relativa, provocan en el animal condiciones de estrés calórico, que afectan el consumo de materia seca, la homeostasis (Herbut \& Angrecka, 2017), la productividad y reproducción de los animales (Krisgnan et al., 2017).

Por tal motivo, el objetivo de este trabajo fue generar modelos matemáticos de predicción de la producción láctea para animales Jersey, que relacionan las condiciones climáticas presentes en el momento que se recolectó la información y las características intrínsecas que presentaban los semovientes en dos sistemas de producción, ubicados a 600 y 1800 m.s.n.m. en la provincia de Cartago, Costa Rica. 


\section{MATERIALES Y MÉTODOS}

Características del estudio: Se utilizó la base de datos desarrollada por Conejo (2017), donde se relacionó de forma retrospectiva la producción de animales Jersey en dos pisos altitudinales con las condiciones climáticas presentes en los años de estudio. Se utilizó la información generada desde el año 2003 al 2014 en el sistema de producción ubicado a 660m.s.n.m. para un total de 61 animales, mientras que en el sistema ubicado a 1800 m.s.n.m., la información se registró entre los años 2011 al 2014, donde se contó con 387 animales. Ambos sistemas se ubicaron en la provincia de Cartago, Costa Rica $\left(9^{\circ} 55^{\prime} 24.36^{\prime \prime} \mathrm{N}-83^{\circ} 51^{\prime} 18.16^{\prime \prime O}\right.$ a 1800 m.s.n.m., y $9^{\circ} 54^{\prime} 13.39^{\prime \prime} \mathrm{N}-83^{\circ} 40^{\prime} 11.37^{\circ} \mathrm{O}$ a $660 \mathrm{~m}$. s.n.m.) (Cuadro 1). Los sistemas de producción presentaron similitud en el manejo de los animales Jersey, donde predominó el pastoreo rotacional, dos ordeños al día, suplementación de alimento balanceado, sal y minerales en canoa previo al ordeño. La información recolectada y organizada en la base de datos vinculo las características temporales, intrínsecas y ambientales, de cada registro de producción, donde todas las semanas durante los rangos descritos en cada localidad, se registró el animal (identificación), edad, número de lactancia, kg de leche producidos el día del registro, días en lactancia, el mes y el año. En el caso de la temperatura, precipitación, radiación, humedad relativa y el índice de humedad y temperatura, se registró el valor presente en las estaciones meteorológicas, presentes en cada sistema de producción, el día de la recolección de la información.

Desarrollo de ecuaciones de predicción de la producción láctea según variables ambientales, intrínsecas y temporales: Se evaluó para cada piso altitudinal, la dependencia de la producción láctea diaria por animal registrada con las variables temporales (año y mes de recolección de la información), variables intrínsecas de los animales evaluados (número de lactancia, edad en semanas y los días de lactancia), y las variables ambientales presentes (temperatura, precipitación, radiación, humedad relativa e índice de humedad-temperatura). Para tal fin, se utilizó el procedimiento STEPWISE de SAS (2012), el cual consiste en realizar una regresión múltiple donde valora la permanencia de cada una de las variables evaluadas en el modelo estadístico.

En esta investigación, se realizaron tres ecuaciones de predicción en cada sistema, bajo la hipótesis de que en los sistemas de producción no se registren todas las variables independientes evaluadas, según el modelo estadístico descrito a continuación:

donde;

$$
Y=U+\beta_{0} A_{i}+\beta_{1} M_{j}+\beta_{2} L_{k}+\beta_{3} E_{l}+\beta_{4} D I_{m}+\beta_{5} T_{n}+\beta_{6} P_{o}+\beta_{7} R_{p}+\beta_{8} H R_{q}+\beta_{g} I T H_{r}+e_{i j k l m n o p q r}
$$

\footnotetext{
$\mathrm{Y}=$ producción de leche diaria en kilogramos;

$U$ = intercepto de la población;

$\beta_{0} A_{i}=$ estimador del efecto del año sobre la producción láctea;

$\beta_{1} M_{j}=$ estimador del efecto del mes sobre la producción láctea;

$\beta_{2} L_{k}=$ estimador del efecto del número de lactancia del animal sobre la producción láctea;

$\beta_{3} E_{1}=$ estimador del efecto de la edad del animal sobre la producción láctea;

$\beta_{4} K_{m}=$ estimador del efecto de los días de lactancia sobre la producción de láctea;

$\beta_{5} \mathrm{DI}_{\mathrm{n}}=$ estimador del efecto de la temperatura diaria sobre la producción láctea;

$\beta_{6} T_{r}=$ estimador del efecto de la precipitación sobre la producción láctea;

$\beta_{7} P_{0}=$ estimador del efecto de la radiación sobre la producción láctea;

$\beta_{8} R_{p}=$ estimador de la humedad relativa sobre la producción láctea;

$\beta_{9} \mathrm{HR}_{\mathrm{q}}=$ estimador del efecto del índice de humedad-temperatura sobre la producción láctea;

$\mathrm{e}_{\mathrm{ijklmnopqr}}=$ efecto residual de las variables independientes.
} 
Con la información de las variables temporales, intrínsecas y ambientales descritas para cada sistema de producción (Cuadro 1), se procedió a sustituir en las ecuaciones resultantes el valor promedio para cada variable, que presentó significancia en el modelo. Para tal fin, se multiplicó el estimador de cada variable determinado en este trabajo con el valor promedio de la misma variable. Por último, la sumatoria de los productos resultantes para cada componente de la ecuación, fue el valor utilizado para estimar la producción láctea promedio del semoviente analizado, en este caso, la población promedio de los animales, condiciones ambientales y temporales.

\section{CUADRO 1}

Condiciones ambientales e indicadores productivos utilizados para el desarrollo de ecuaciones de predicción de la producción láctea de animales Jersey ubicados a 660 y 1 800m.s.n.m., Cartago, Costa Rica, 2016

\begin{tabular}{|c|c|c|c|c|c|}
\hline \multirow{2}{*}{ Variables } & \multicolumn{2}{|c|}{ Número observaciones evaluadas } & \multirow{2}{*}{ Unidades } & \multicolumn{2}{|c|}{ Valor promedio } \\
\hline & 600m.s.n.m. & 1800 m.s.n.m. & & 600m.s.n.m. & 1800 m.s.n.m. \\
\hline \multicolumn{6}{|l|}{ Temporales } \\
\hline Año & 12089 & 14641 & Años & 12 & 5 \\
\hline Mes & 12089 & 14641 & Meses & 12 & 12 \\
\hline \multicolumn{6}{|l|}{ Intrínsecas } \\
\hline Lactación & 12089 & 14641 & Cantidad & $2,97 \pm 1,96$ & $3,13 \pm 2,04$ \\
\hline Edad & 12089 & - & Meses & $61,72 \pm 27,12$ & - \\
\hline Kilos de leche & 12083 & 13820 & $\mathrm{Kg}$ & $15,39 \pm 4,11$ & $20,02 \pm 4,93$ \\
\hline Días de lactación & 12089 & 14639 & Días & $183,25 \pm 122,42$ & $138,64 \pm 87,21$ \\
\hline \multicolumn{6}{|l|}{ Ambientales } \\
\hline Temperatura & 12089 & 13722 & ${ }^{\circ} \mathrm{C}$ & $22,27 \pm 1,30$ & $15,09 \pm 1,17$ \\
\hline Precipitación & 12089 & 13723 & mm/día & $7,65 \pm 19,01$ & $1,97 \pm 4,53$ \\
\hline Radiación & 12089 & 13722 & $\mathrm{Mj} / \mathrm{m}^{2}$ & $15,98 \pm 5,80$ & $20,85 \pm 11,94$ \\
\hline Humedad relativa & 12089 & 13722 & $\%$ & $90,56 \pm 5,84$ & $80,73 \pm 9,97$ \\
\hline $\mathrm{ITH} \mathrm{H}^{*}$ & 12089 & 13722 & & $71,54 \pm 2,18$ & $59,54 \pm 1,88$ \\
\hline
\end{tabular}

*índice humedad y temperatura

Ética, conflicto de intereses y declaración de financiamiento: Los autores declaran haber cumplido con todos los requisitos éticos y legales pertinentes, tanto durante el estudio como en el manuscrito; que no hay conflictos de interés de ningún tipo, y que todas las fuentes financieras se detallan plena y claramente en la sección de agradecimientos. Asimismo, están de acuerdo con la versión editada final del documento. El respectivo documento legal firmado se encuentra en los archivos de la revista.

\section{RESULTADOS}

Rango de las condiciones ambientales, según la altura de referencia de los sistemas: Los datos registrados de las variables ambientales e intrínsecas en cada localidad presentaron un rango diferente según sus valores extremos, variación que permite, que las ecuaciones que se desarrollan a partir de la información recopilada, se asocie a los valores mínimos y máximos; no así, de forma estricta a la altura del sistema de producción. La temperatura en ambas localidades permite una fluctuación promedio de los datos de 2,60 y $2,34^{\circ} \mathrm{C}$ para las localidades a 600 y $1800 \mathrm{~m}$. .n.m. respectivamente (Cuadro 1). En el caso de la precipitación, radiación, humedad relativa y el índice de humedad y temperatura, estos valores son de 26,66 y $6,50 \mathrm{~mm} /$ día; 11,60 y $23,88 \mathrm{Mj} / \mathrm{m}^{2} ; 11,68$ y $19,94 \%$; y 4,36 y 3,76; para las localidades a 600 y 1800 m.s.n.m. respectivamente. 
Ecuación de estimación según variables intrínsecas, temporales y ambientales: Se determinaron tres variables intrínsecas, una temporal y cuatro ambientales que presentaron significancia para estimar la producción de leche de animales Jersey ubicados a 660m.s.n.m. (Cuadro 2). Se observó que, a mayor edad del animal, precipitación, radiación, índice de humedad relativa y temperatura se presenta un efecto positivo sobre la producción de leche (Fig.1). Mientras que, conforme avanza el mes de evaluación, el número de lactancia y los días de lactancias; se incrementa la temperatura y la humedad relativa, la productividad del animal tiende a disminuir (Fig.1).

\section{CUADRO 2}

Variables utilizadas para el desarrollo de la ecuación de estimación de la producción de leche de animales Jersey ubicados 660m.s.n.m., Turrialba, Cartago, Costa Rica, 2016

\begin{tabular}{lccccc}
\hline \multicolumn{1}{c}{ Variable } & $\begin{array}{c}\text { Estimador del } \\
\text { parámetro }\end{array}$ & $\begin{array}{c}\text { Error } \\
\text { estándar }\end{array}$ & Tipo II SS & F-Valor & Pr>F \\
\hline Interceptor & $-59,73903$ & 8,204 & 586,08 & 53,02 & $<0,0001$ \\
Mes & $-0,13990$ & 0,009 & 2421,07 & 219,03 & $<0,0001$ \\
N $^{\circ}$ lactancia & $-0,98812$ & 0,068 & 2335,49 & 211,28 & $<0,0001$ \\
Edad & 0,08370 & 0,005 & 3031,09 & 274,21 & $<0,0001$ \\
Días de lactancia & $-0,02143$ & 0,000 & 52942 & 4789,43 & $<0,0001$ \\
Temperatura & $-4,52292$ & 0,516 & 849,41 & 76,84 & $<0,0001$ \\
Precipitación & 0,00376 & 0,002 & 54,52 & 4,93 & 0,0264 \\
Radiación & 0,04428 & 0,007 & 466,27 & 42,18 & $<0,0001$ \\
Humedad relativa & $-0,15629$ & 0,021 & 582,85 & 52,73 & $<0,0001$ \\
IHT & 2,68223 & 0,300 & 880,84 & 79,69 & $<0,0001$ \\
\hline
\end{tabular}

*índice humedad y temperatura

La ecuación resultante para estimar la producción de leche en animales Jersey (kg/animal/día) que se encuentren ubicados a una altura de 660m.s.n.m., presenta los siguientes componentes:

\footnotetext{
$\mathrm{Y}=-59,73903+(-0,13990 *$ mes $)+\left(-0,98812 * \mathrm{~N}^{\circ}\right.$ de lactación $)+(0,08370 *$ edad $)+(-0,02143 *$ días de lactancia $)+(-$ $4,52292 *$ temperatura ambiental $)+(0,00376 *$ precipitación $)+(0,04428 *$ radiación $)+(-0,15629 *$ humedad relativa $)+(2,68223 * \mathrm{IHT})$
}

En el caso de animales Jersey en sistemas de producción ubicados a 1800 m.s.n.m., se encontró que aumentos en el número de lactancia, el mes, y la precipitación favorecen incrementos en la producción de leche diaria de los semovientes (Cuadro 3 y Fig. 1). La ecuación estimada en estos sistemas, se describe de la siguiente manera:

\footnotetext{
$\mathrm{Y}=27,50154+\left(0,57339 * \mathrm{~N}^{\circ}\right.$ de lactancias $)+(0,02393 * \mathrm{mes})+(-0,03188 *$ días de lactancia $)+(-0,06494 *$ radiación $)+(-$
} $0,04206 *$ humedad relativa $)+(0,01775 *$ precipitación $)$ 


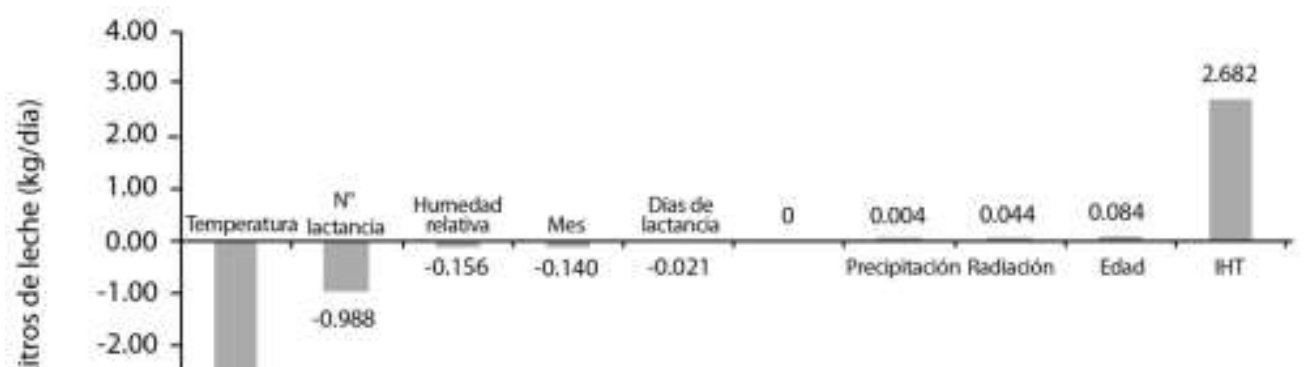

$-3.00$

$-4.00$

$-5.00-4.523$

Altura 600 m.s.n.m.

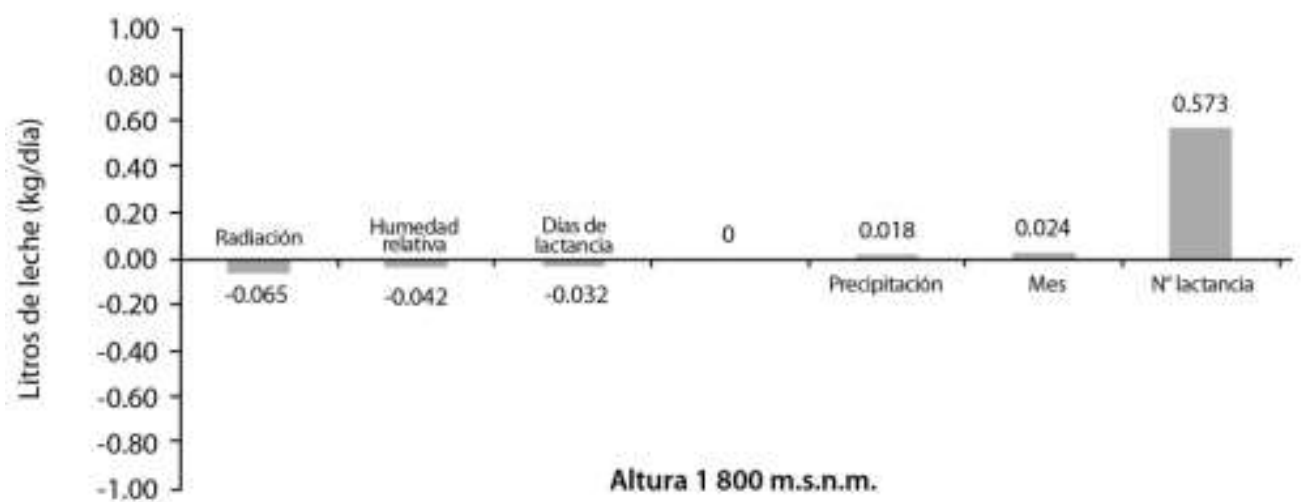

Fig 1. Organización de las variables temporales, intrínsecas y ambientales según la magnitud de afectación en la producción de leche diaria de animales Jersey ubicados a 600 y 1800 m.s.n.m. Cartago, 2016.

Con base en la altura a la cual se encuentran los semovientes, la variable evaluada presentó un efecto inverso en la producción láctea diaria, como lo fue el número de lactancia y el mes, según el sistema evaluado (Fig. 1).

\section{CUADRO 3}

Variables utilizadas para el desarrollo de la ecuación de estimación de la producción de leche de animales Jersey ubicados 1800 m.s.n.m. Santa Rosa de Oreamuno, Cartago, Costa Rica, 2016

\begin{tabular}{lccccc}
\hline \multicolumn{1}{c}{ Variable } & $\begin{array}{c}\text { Estimador del } \\
\text { parámetro }\end{array}$ & $\begin{array}{c}\text { Error } \\
\text { estándar }\end{array}$ & Tipo II SS & F-Valor & Pr>F \\
\hline Interceptor & 27,50154 & 0,356 & 90329 & 5958,89 & $<0,0001$ \\
N $^{\circ}$ lactancia & 0,57339 & 0,017 & 175220 & 1155,75 & $<0,001$ \\
Mes & 0,02393 & 0,010 & 78,45 & 5,18 & 0,0229 \\
Días de lactancia & $-0,03188$ & 0,000 & 94677 & 6245,71 & $<0,0001$ \\
Radiación & $-0,06494$ & 0,003 & 5931,99 & 391,32 & $<0,0001$ \\
Humedad relativa & $-0,04206$ & 0,003 & 1757,34 & 115,93 & $<0,0001$ \\
Precipitación & 0,01775 & 0,008 & 75,40 & 4,97 & 0,0257 \\
\hline
\end{tabular}


Ecuación de estimación según variables intrínsecas: Dentro de las variables intrínsecas o propias del animal, se desarrolló un modelo para determinar la producción láctea para los sistemas de producción que no presenten información meteorológica actualizada. Se determinaron tres variables en cada sistema, que presentaron significancia en la producción de leche de los semovientes, las cuales fueron utilizadas para el desarrollo del modelo matemático (Cuadro 4 y Cuadro 5). Se describen los efectos negativos en la producción de leche, cuando se incrementa el número de lactancias y los días de lactancia en la ubicación a 660m.s.n.m., según la ecuación de estimación descrita en el Cuadro 3, la cual se describe a continuación.

$Y=17,23408+\left(-1,03029 * N^{\circ}\right.$ de lactancia $)+(0,08501 *$ edad $)+(-0,02196 *$ días de lactancia $)$

\section{CUADRO 4}

Variables intrínsecas utilizadas para el desarrollo de la ecuación de estimación de la producción de leche de animales Jersey ubicados a 660m.s.n.m. Turrialba, Cartago, Costa Rica, 2016

\begin{tabular}{lccccc}
\hline \multicolumn{1}{c}{ Variable } & $\begin{array}{c}\text { Estimador del } \\
\text { parámetro }\end{array}$ & $\begin{array}{c}\text { Error } \\
\text { estándar }\end{array}$ & Tipo II SS & F-Valor & Pr>F \\
\hline Interceptor & 17,23408 & 0,108 & 286678 & 25189,2 & $<0,0001$ \\
N $^{\circ}$ lactancia & $-1,03029$ & 0,069 & 2544,71 & 223,59 & $<0,0001$ \\
Edad & 0,08501 & 0,005 & 3133,24 & 275,31 & $<0,0001$ \\
Días de lactancia & $-0,02196$ & 0,000 & 56219 & 4939,72 & $<0,0001$ \\
\hline
\end{tabular}

\section{CUADRO 5}

Variables intrínsecas utilizadas para el desarrollo de la ecuación de estimación de la producción de leche de animales Jersey ubicados a 1 800m.s.n.m. Santa Rosa de Oreamuno, Cartago, Costa Rica, 2016

\begin{tabular}{lccccc}
\hline \multicolumn{1}{c}{ Variable } & $\begin{array}{c}\text { Estimador del } \\
\text { parámetro }\end{array}$ & $\begin{array}{c}\text { Error } \\
\text { estándar }\end{array}$ & Tipo II SS & F-Valor & Pr>F \\
\hline Interceptor & 22,20999 & 0,104 & 709021 & 45382,90 & $<0,0001$ \\
$\mathrm{~N}^{\circ}$ lactancia & 0,58015 & 0,016 & 19156 & 1226,15 & $<0,0001$ \\
Mes & 0,08774 & 0,009 & 1352,54 & 86,57 & $<0,0001$ \\
Días de lactancia & $-0,03172$ & 0,000 & 101103 & 6471,35 & $<0,0001$ \\
\hline
\end{tabular}

Los días de lactancia presentaron un efecto al disminuir la producción en animales ubicados a 1800 m.s.n.m., donde por cada día acumulado, se redujo en 0,03L la productividad del semoviente (Cuadro 5). Según el resultado obtenido en el Cuadro 5, la ecuación de estimación es la siguiente: $\mathrm{Y}=22,20999+\left(0,58015^{*} \mathrm{~N}^{\circ}\right.$ de lactancia $)+(0,08774 *$ mes $)+(-0,03172 *$ días de lactancia $)$

Ecuación de estimación según variables ambientales: En el caso del sistema de producción a 660m.s.n.m., incrementos en la temperatura y la humedad relativa del sistema afectan de forma negativa la productividad de los animales, en cambio, la productividad del animal se favorece con incrementos en la radiación, precipitación y el IHT (Cuadro 6). La ecuación de estimación se resume de la siguiente manera:

$$
\begin{aligned}
Y=-86,05046+(-6,47262 * \text { temperatura })+ & (0,00314 * \text { precipitación })+(0,07518 * \text { radiación })+(-0,22944 * \text { humedad } \\
& \text { relativa })+(3,70597 * \mathrm{HT})
\end{aligned}
$$




\section{CUADRO 6}

Variables ambientales utilizadas para el desarrollo de la ecuación de estimación de la producción de leche de animales Jersey ubicados a 660m.s.n.m. Turrialba, Cartago, Costa Rica, 2016

\begin{tabular}{lccccc}
\hline \multicolumn{1}{c}{ Variable } & $\begin{array}{c}\text { Estimador del } \\
\text { parámetro }\end{array}$ & $\begin{array}{c}\text { Error } \\
\text { estándar }\end{array}$ & Tipo II SS & F-Valor & Pr>F \\
\hline Intercepto & $-86,05046$ & 9,967 & 1246,66 & 74,54 & $<0,0001$ \\
Temperatura ambiental & $-6,47262$ & 0,629 & 1767,95 & 105,71 & $<0,0001$ \\
Precipitación & 0,00314 & 0,002 & 37,95 & 2,27 & 0,1320 \\
Radiación & 0,07518 & 0,008 & 1408,85 & 84,24 & $<0,0001$ \\
Humedad relativa & $-0,22944$ & 0,026 & 1266,84 & 75,75 & $<0,0001$ \\
IHT* $^{*}$ & 3,70597 & 0,366 & 1712,52 & 102,39 & $<0,0001$ \\
\hline
\end{tabular}

*Índice humedad y temperatura

Aumentos en la radiación solar, la humedad relativa y el IHT mermaron la productividad de los animales, siendo este último el de mayor impacto, con una merma de $1,5 \mathrm{~L}$ de leche por día en el sistema a 1800 m.s.n.m., mientras que un incremento en la temperatura favoreció la productividad de los animales (Cuadro 7). Según el análisis de la información y los resultados descritos, se resume la ecuación de predicción en este sistema:

$\mathrm{Y}=72,97387+(-0,06644 *$ radiación $)+(-0,01690 *$ humedad relativa $)+(2,67970 *$ temperatura ambiental $)+(-1,52592 * \mathrm{IHT})$

\section{CUADRO 7}

Variables ambientales utilizadas para el desarrollo de la ecuación de estimación de la producción de leche de animales Jersey ubicados a 1800 m.s.n.m. Santa Rosa de Oreamuno, Cartago, Costa Rica, 2016

\begin{tabular}{lccccc}
\hline \multicolumn{1}{c}{ Variable } & $\begin{array}{c}\text { Estimador del } \\
\text { parámetro }\end{array}$ & $\begin{array}{c}\text { Error } \\
\text { estándar }\end{array}$ & Tipo II SS & F-Valor & Pr $>$ F \\
\hline Intercepto & 72,97387 & 10,746 & 1093,03 & 46,11 & $<0,0001$ \\
Radiación & $-0,06644$ & 0,004 & 7004,45 & 295,49 & $<0,0001$ \\
Humedad relativa & $-0,01690$ & 0,005 & 231,54 & 9,77 & 0,0018 \\
Temperatura ambiental & 2,67970 & 0,506 & 663,41 & 27,99 & $<0,0001$ \\
IHT & $-1,52592$ & 0,311 & 570,29 & 24,06 & $<0,001$ \\
\hline
\end{tabular}

*Índice humedad y temperatura

Uso de las ecuaciones desarrolladas para la estimación de la producción láctea de animales Jersey a dos pisos altitudinales: De los tres modelos desarrollados, las ecuaciones que utilizan las variables temporales, ambientales e intrínsecas del animal permiten estimar la producción del semoviente con una diferencia de 0,50 y $0,21 \mathrm{~kg}$ de leche para los sistemas de producción a 600 y 1800 m.s.n.m. respectivamente (Cuadro 8). En el caso de las variables ambientales, con la estimación por medio de las ecuaciones generadas, las variaciones fueron de 0,01 y $0,21 \mathrm{~kg}$ de leche a los 600 y $1800 \mathrm{~m}$. s.n.m. respectivamente. Mientras que, en el caso de los modelos que solo utilizan las variables intrínsecas de los semovientes, no se presentaron fluctuaciones en la producción láctea promedio en los $600 \mathrm{~m} . s . n . m$ y 0,12kg a los 1800 m.s.n.m. 


\section{CUADRO 8}

Producción láctea estimada de animales Jersey, según las ecuaciones de estimación desarrolladas que utilizan las variables temporales, ambientales e intrínsecas a dos altitudes en la provincia de Cartago. Costa Rica, 2018

\begin{tabular}{|c|c|c|c|}
\hline & $\begin{array}{c}\begin{array}{c}\text { Estimador de la } \\
\text { variable }\end{array} \\
\end{array}$ & Valor promedio & $\begin{array}{c}\text { Parámetro*Valor } \\
\text { promedio }\end{array}$ \\
\hline \multicolumn{4}{|c|}{ Variables para el sistema a 600m.s.n.m. } \\
\hline Intercepto & $-59,73903$ & & $-59,74$ \\
\hline Mes & $-0,1399$ & 3 & $-0,42$ \\
\hline $\mathrm{N}^{\circ}$ lactancia & $-0,98812$ & 2,97 & $-2,93$ \\
\hline Edad & 0,0837 & 61,72 & 5,17 \\
\hline Días de lactancia & $-0,02143$ & 183,25 & $-3,93$ \\
\hline Temperatura & $-4,52292$ & 22,27 & $-100,73$ \\
\hline Precipitación & 0,00376 & 7,65 & 0,03 \\
\hline Radiación & 0,04428 & 15,98 & 0,71 \\
\hline Humedad relativa & $-0,15629$ & 90,56 & $-14,15$ \\
\hline \multirow[t]{2}{*}{$\mathrm{IHT}$} & 2,68223 & 71,54 & 191,89 \\
\hline & \multicolumn{2}{|c|}{ Producción de leche estimada ( $\Sigma$ ) } & 15,89 \\
\hline \multicolumn{4}{|c|}{ Variables para el sistema a 1800 m.s.n.m. } \\
\hline Intercepto & 27,50154 & & 27,50 \\
\hline $\mathrm{N}^{\circ}$ lactancia & 0,57339 & 3,13 & 1,79 \\
\hline Mes & 0,02393 & 3 & 0,07 \\
\hline Días de lactancia & $-0,03188$ & 138,64 & $-4,42$ \\
\hline Radiación & $-0,06494$ & 20,85 & $-1,35$ \\
\hline Humedad relativa & $-0,04206$ & 80,73 & $-3,40$ \\
\hline \multirow[t]{2}{*}{ Precipitación } & 0,01775 & 1,97 & 0,03 \\
\hline & \multicolumn{2}{|c|}{ Producción de leche estimada $(\Sigma)$} & 20,23 \\
\hline
\end{tabular}

\section{DISCUSIÓN}

De las variables ambientales, temporales e intrínsecas evaluadas, se determinaron efectos positivos y negativos sobre el semoviente que afectaron su productividad durante la lactancia. Además, según la altura en que se desarrolló la actividad, se observó una dirección y magnitud diferente del efecto sobre la productividad medida en kilogramos de leche al día para una misma variable. Situación que se asocia al rango de la variable que se determinó en cada sistema productivo, lo que permite generar microclimas según la ubicación del sistema (MINAET/IMN/PNUD/CRRH, 2008). Aunque en esta investigación no se consideró el efecto en los componentes de la leche, se describe en la literatura que además de una merma en la producción de leche, se disminuyen sus componentes, lo cual afecta el ingreso económico del sistema productivo (Echeverri \& Restrepo, 2009).

En el caso del índice de humedad y temperatura utilizado en este trabajo, este indicador presentó una diferencia de 12,05 unidades entre sistemas, variación que se obtuvo de valores mayores de temperatura y humedad relativa en zonas de menor altura, y en zonas de mayor altura con menores registros de humedad relativa y temperatura. Se sugiere que esta diferencia generó una reducción en el consumo de materia seca, mayor consumo de agua, cambios en la tasa metabólica, incremento de las pérdidas de humedad por evaporación, incremento en la tasa respiratoria y temperatura rectal en los animales a 600m.s.n.m. (Ghosh, Kesh, Tudu, \& Datta, 2017), lo que generó un efecto negativo en los kilogramos de leche producida en relación a las condiciones 
del sistema de producción a 1800 m.s.n.m., donde se favoreció la producción (Herbut \& Angrecka, 2017). Este comportamiento se podría asociar a condiciones climáticas cercanas a las condiciones de termorregulación del Bos taurus (Garner et al., 2016), y a un proceso de adaptación de los animales desde su nacimiento (WingChing-Jones et al., 2008).

En el caso de la edad del semoviente, el número de lactancia evaluada y los días de lactancia, son variables que se asocian en una primera etapa con la madurez del animal al alcanzar su peso adulto, con procesos de adaptación a situaciones ambientales, sanitarias, de manejo y nutricionales; y de menos desgaste del organismo por el padecimiento de estas situaciones, que le permite a los semovientes mantener una condición corporal entre ciclos productivos apropiada para optimizar la producción (Eastham et al., 2018). En cambio, una segunda etapa se presenta a mayor edad o permanencia en el sistema, donde los animales registran mayor cantidad de eventos relacionados a enfermedades metabólicas (Saborío-Montero, Vargas-Leitón, Romero-Zuñiga, \& Sánchez, 2017), podales (Solano et al., 2016), salud de ubre (Mora, Vargas, Moreno, \& Camacho, 2015), sanitarias, reproductivas (Daros, Hotzel, Bran, LeBlanc, \& von Keyserlingk, 2017) y lesiones por mal manejo, que desgastan al organismo y provocan de forma diferente una disminución en la capacidad productiva de cada animal. Esta respuesta de los animales a diferentes condiciones podría estar asociada a la implementación de programas de inseminación artificial y al uso de material genético (semen y embriones), que permitía de forma rápida incluir genes de diferentes ambientes, lo que aumenta la biodiversidad genética y la capacidad de interacción con el ambiente (Garner et al., 2016).

Los días de lactancia de los semovientes afectaron la producción láctea sin importar la ubicación del sistema, debido a que por la naturaleza de la lactancia se parte de un valor de producción establecido por el potencial genético y su interacción con el ambiente al momento del parto, hasta alcanzar un punto máximo, donde inicia una disminución paulatina en la producción láctea marcada por la persistencia hasta el momento del secado (Valerin, 1997).

De las variables temporales, el mes de evaluación presentó un efecto diferente según el sistema evaluado, donde conforme avanza el año, el efecto fue positivo a mayor altura y negativo a menor altitud para la producción de leche diaria. Aunque ambas zonas presentan influencia del Caribe, la distribución de las lluvias a través del año provoca un efecto indirecto en la producción de forraje, que afecta el aporte de nutrimentos mediante el consumo de materia seca. En condiciones de baja precipitación, en zonas de menor altura, se reduce el crecimiento de forraje por disminución del agua precipitada y aumento de la radiación, condiciones que afectan el crecimiento de la planta (Pezzopane et al., 2016). En el caso contrario, este mismo comportamiento permite que los forrajes en altura presenten un mejor desarrollo, debido a que se reduce la nubosidad y aumenta la fotosíntesis (Eriksen \& Whitney, 1981). Sin embargo, el incremento en la radiación sobre el animal genera un aumento de la temperatura corporal que provoca en los animales manejados en zonas de altura, estrés calórico (Berman, 2011). Esta misma condición, sumada a una distribución uniforme de las lluvias durante el año, genera en zonas cercanas a los 660 m s.n.m. un mejor crecimiento del forraje, que, asociado a un proceso de adaptación de los animales desde su nacimiento, permite una combinación de variables que favorecen las condiciones para que los animales aumenten su producción (Fanta, 2017).

El conocer el impacto de las variables evaluadas sobre la producción láctea de los semovientes, es una herramienta que podría permitir al sistema productivo implementar los cambios o modificar las prácticas de manejo para favorecer las condiciones de bienestar animal (von Keyserlingj, Rushen, de Passillé, \& Weary, 2009), y optimizar el uso de los recursos bajo la relación suelo, cultivo, animal y ambiente (Sizemore, 2015). En relación a los sistemas productivos presentes en Costa Rica, Vargas-Leitón, Solís-Guzmán, Sáenz-Segura y León-Hidalgo (2013), describen cinco tipos de sistemas productivos de lechería especializada según la altura, donde los dos sistemas 
evaluados en este trabajo pertenecen a lechería especializada intensiva de altura y lechería especializada intensiva de bajura.

\section{AGRADECIMIENTOS}

Agradecemos el apoyo de José Joaquín Jiménez Zamora por el apoyo brindado en el desarrollo de esta investigación, y a los señores Roberto Ugalde y Oscar Garita, por su dedicación en sus funciones en el Módulo Lechero de la Universidad de Costa Rica.

\section{REFERENCIAS}

Berman, A. (2011). Invited review: Are adaptations present to support dairy cattle in warm climates. Journal Dairy Science, 94, 2147-2158. DOI: 10.3168/jds.2010-3962

Cascante, A. (2008). Efecto de la edad a primer parto sobre algunas variables productivas, primer intervalo entre partos y características de la curva de lactancia de vacas lecheras en la zona norte de Costa Rica (Tesis inédita de licenciatura). Universidad de Costa Rica, San José, Costa Rica.

Castello-Umaña, M., Alpizar-Naranjo, A., Padilla-Fallas, J., \& Keim-San Martin, J. (2017). Efecto de la edad a primer servicio, número y época de parto sobre el comportamiento de la curva de lactancia en vacas Jersey. Nutrición Animal Tropical, 11(2), 1-22. DOI: 10.15517/nat.v1112.31306

Castillo-Badilla, G., Salazar-Carranza, M., Murillo-Herrera, J., \& Romero-Zúñiga, J. (2013). Efecto de edad a primer parto sobre parámetros productivos en vacas Jersey de Costa Rica. Agronomía Mesoamericana, 24, 177-187. DOI: $10.15359 /$ rcv.33-1.2

Cole, J. B., Ehrlich, J. L., \& Null, D. J. (2012). Short communication: Projenting milk yield using best prediction and the MilBot lactation model. Journal Dairy Science, 95, 4041-4044. DOI: 10.3168/jds.2011-4905

Conejo, J. F. (2017). Efectos de las condiciones ambientales sobre la producción láctea de dos hatos de Ganado Jersey en dos pisos altitudinales de la provincia de Cartago (Tesis inédita de licenciatura) Universidad de Costa Rica, San José, Costa Rica.

Cuevas, V., Loaiza, A., Astengo, H., Moreno, T., Borja, M., Reyes, J., \& González, D. (2018). Análisis de la función de producción de leche en el sistema bovimos doble proposito en Ahome, Sinaloa. Revista Mexica de Ciencias Pecuarias, 9, 376-386. DOI: 10.22319/rmcp.v9i2.4545

Daros, R., Hotzel, M., Bran, J., LeBlanc, S., \& von Keyserlingk, M. (2017). Prevalence and risk factors for transition period diseases in grazing dairy cows in Brasil. Preventive Veterinary Medicine, 145, 16-22. DOI: 10.1016/j.prevetmed.2017.06.004

Eastham, N., Coates, A., Cripps, P., Richardson, H., Smith R., \& Oikonomou, G. (2018). Associations between age at first calving and subsequent lactation performance in UK Holstein and Holstein-Friesian dairy cows. PloS ONE, 13(6), e0197764. DOI: 10.1371/journal.pone.0197764

Echeverri, J. J., \& Restrepo, L. F. (2009). Efecto meteorológico sobre la producción y calidad de la leche en dos municipios de Antioquia, Colombia. Revista Lasallista de Investigación, 6(1), 50-57.

Eriksen, F. I., \& Whitney, A. S. (1981). Effects of light intensity on growth of some tropical forage species. I. Interaction of light intensity and nitrogen fertilization on six forage grasses. Agronomy Journal, 73, 427-433. DOI: 10.2134/agronj1981.00021962007300030011x

Fanta, M. (2017). Physiological adaptation of Holstein Frisian dairy cattle in Ethiopia: Review article. Journal of Biology Agriculture Healthcare, 7(13), 67-78. 
Garner, J. B., Douglas, M. L., Williams, S. R., Wales, W. J., Marett, L. C., Nguyen, T. T., ... Hayes, B. J. (2016). Genomic selection improves heat tolerance in dairy cattle. Scientific Reports, 6, 34114. DOI: 10.1038/srep34114

Ghosh, C.P., Kesh, S. S., Tudu, N. K., \& Datta, S. (2017). Heat stress in dairy animals-Its impact and remedies: A review. International Journal of Pure \& Applied Bioscience, 5, 953-965. DOI: 10.18782/2320-7051.2577

Hagnestam-Nielsen, C., Emanuelson, U., Berglund, B., \& Strandberg, E. (2009). Relationship between somatic cell count and milk yield in different stages of lactation. Journal of Dairy Science, 92, 3124-3133. DOI: 10.3168/jds.2008-1719

Herbut, P., \& Angrecka. S. (2017). Relationship between THI level and dairy cows' behavior during summer period. Italian Journal of Animal Science, 17, 226-233. DOI: 10.1080/1828051X.2017.1333892

Klopcic, M., Koops, W. J., \& Kuipers. A. (2013). Technical note: A mathematical function to predict daily milk yield of dairy cows in relation to the interval between milkings. Journal of Dairy Science, 96, 6084-6090. DOI: 10.3168/jds.20126391

Krishnan, G., Bagath, M., Pragna, P., Kusha, M., Aleena, J., Ravindranathan, P., ... Bhatta, B. (2017). Mitigation of the heat stress impact in livestock reproduction. In R. Payan (Ed.), Theriogenology (pp. 63-89). London, UK: In Tech Open. DOI: 10.5772/intechopen.69091

Krizsan, S. J., Sairanen, A., Hojer, A., \& Huhtanen, P. (2014). Evaluation of different feed intake models for dairy cows. Journal of Dairy Science, 97, 2387-2397. DOI: 10-3168/jds.2013-7561

MINAET (Ministerio del Ambiente Energía y Telecomunicaciones), IMN (Instituto Meteorológico Nacional), PNUD, y CRRH (Comité Regional de Recursos Hidráulicos). (2008). El clima, su variabilidad y cambio climático en Costa Rica. San José, Costa Rica: MINAET, IMN, PNUD, y CRRH.

Molina, J., \& Boschini, C. (1979). Ajuste de la curva de lactancia de ganado Holstein con un modelo lineal modal. Agronomía Costarricense, 3(2), 167-174.

Mora, M., Vargas, B., Moreno, J. J., \& Camacho, J. (2015). Factores de riesgo para la incidencia de mastitis clínica en ganado lechero de Costa Rica. Agronomía Costarricense, 39(2), 77-89.

Murphy, M. D., O’Mahony, M. J., Shalloo, L., French, P., \& Upton, J. (2014). Comparison of modeling techniques for milkproduction forecasting. Journal of Dairy Science, 97, 3352-3363. DOI: 10.3168/jds.2013-7451

Pezzopane J., Santos, P., Evangelista, S., Bosi, C., Cavalcantes, A., Bettiol, G., ... Pellegrino, G. (2016). Panicum maximum cv. Tanzania: climate trends and regional pasture production in Brazil. Grass and Forage Science, 72(1), 104-117. DOI: $10.1111 / g f s .12229$

Quintero, J., Serna, J., Hurtado, N., Rosero, R., \& Cerón-Muñoz. M. (2007). Modelos matemáticos para curvas de lactancia en ganado lechero. Revista Colombiana de Ciencias Pecuarias, 20, 149-156.

Roche, J. R., Friggens, N. C., Kay, J. K., Fisher, M. W., Stafford, K. J., \& Beery, D. P. (2009). Invited review: Body condition score and its association with dairy cow productivity, health, and welfare. Journal of Dairy Science, 92, 5769-5801. DOI: $10.3168 /$ jds.2009.2009-2431

Rodríguez, J. G., Olivares, J. L., Sánchez, Y., Alemán, Y., \& Arece. J. (2013). Cambios climáticos y su efecto sobre algunos grupos de parásitos. Revista de Salud Animal, 35(3), 145-150.

Saborío-Montero, A., Vargas-Leitón, B., Romero-Zuñiga, J. J., \& Sánchez, J. (2017). Risk factor associated with milk fever occurrence in grazing dairy cattle. Journal of Dairy Science, 100, 1-8. DOI: 10.3168/jds.2017-13065

Sánchez, J., \& Soto, H. (1999). Estimación de la calidad nutricional de los forrajes del cantón de San Carlos. Energía para la producción de leche. Nutrición Animal Tropical, 5(1), 31-49.

SAS Institute. (2012). SAS/STAT ${ }^{\circledR 9,4}$ User's Guide. SAS Institute Inc., Cary, NC, USA. 
Sizemore, G. (2015). Accounting for biodiversity in the dairy industry. Journal Environmental Management, 155, $145-153$. DOI: 10.1016/j.jenvman.2015.03.015

Solano, L., Barkema, H. W., Mason, S., Pajor, E. A., LeBlanc, S. J., \& Orsel, K. (2016). Prevalence and distribution of foot lesions in dairy cattle in Alberta, Canada. Journal of Dairy Science, 99, 6828-6841. DOI: 10.3168/jds.2016-10941

Valerin, J. (1997). Caracterización fenotípica y evaluación genética de reproductores Jersey para producción láctea, días abiertos, intervalo entre partos y edad al primer parto (Tesis inédita de licenciatura), Universidad de Costa Rica, San José, Costa Rica.

Vargas-Leitón, B. \& Solano-Patiño, C. (1995). Factores de proyección y de corrección para producción por lactancia en vacas lecheras de Costa Rica. Archivos Latinoamericanos de Producción Animal, 3(2), 149-164.

Vargas-Leitón, B., Solís-Guzmán, O., Sáenz-Segura, F., \& León-Hidalgo, H. (2013). Caracterización y clasificación de hatos lecheros en Costa Rica mediante análisis multivariado. Agronomía Mesoamericana, 24,257-275. DOI: 10.15517/am.v24i2.12525

Von-Keyserlingj, M. A., Rushen, J., de Passillé, A. M., \& Weary, D. M. (2009). Invited review: The welfare of dairy cattleKey concepts and the role of science. Journal of Dairy Science, 92, 4101-4111. DOI: 10.3168./jds.2009-2326

WingChing-Jones, R., Cabalceta-Aguilar, G., \& Alvarado-Hernández, A. (2009). Impacto del pastoreo con ganado Holstein y Jersey sobre la densidad aparente de un andisol. Agronomía Mesoamericana, 20,371-379. DOI: 10.15517/am.v20i2.4953

WingChing-Jones, R., Pérez, R., \& Salazar. E. (2008). Condiciones ambientales y producción de leche de un hato de ganado Jersey en el trópico Húmedo: El caso del Módulo Lechero-SDA/UCR. Agronomía Costarricense, 32(1), 87-94.

Xue, B., Yan,T., Ferris, C. F., \& Mayne, C. S. (2011). Milk production and energy efficiency of Holstein and Jersey-Holstein crossbred dairy cows offered diets containing grass silage. Journal of Dairy Science, 94, 1455-1464. DOI: $10.3168 /$ jds.2010-3663 